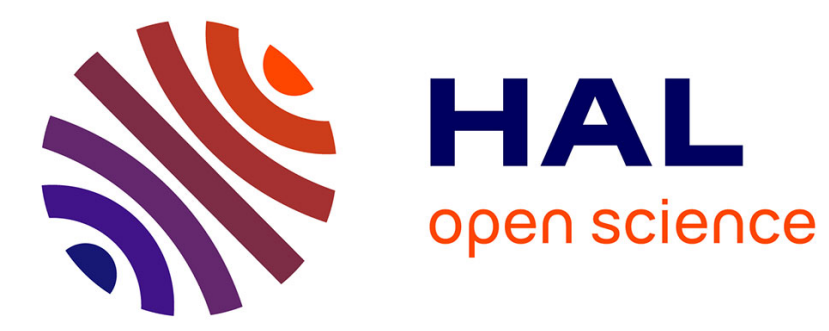

\title{
On the concept of metadislocations in complex metallic alloys
}

\author{
M. Feuerbacher, Marc Heggen
}

\section{To cite this version:}

M. Feuerbacher, Marc Heggen. On the concept of metadislocations in complex metallic alloys. Philosophical Magazine, 2006, 86 (06-08), pp.935-944. 10.1080/14786430500263439 . hal-00513582

\section{HAL Id: hal-00513582 \\ https://hal.science/hal-00513582}

Submitted on 1 Sep 2010

HAL is a multi-disciplinary open access archive for the deposit and dissemination of scientific research documents, whether they are published or not. The documents may come from teaching and research institutions in France or abroad, or from public or private research centers.
L'archive ouverte pluridisciplinaire HAL, est destinée au dépôt et à la diffusion de documents scientifiques de niveau recherche, publiés ou non, émanant des établissements d'enseignement et de recherche français ou étrangers, des laboratoires publics ou privés. 


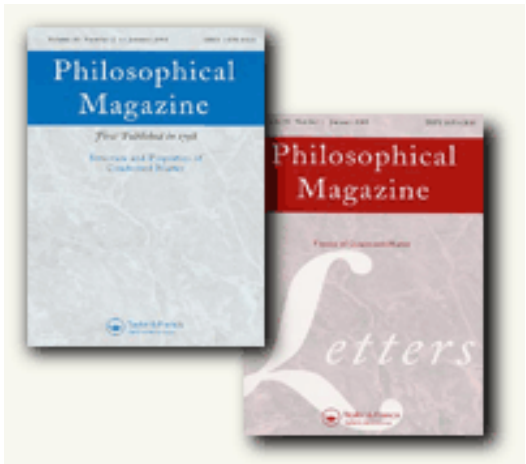

\section{On the concept of metadislocations in complex metallic alloys}

\begin{tabular}{|r|l|}
\hline Journal: & Philosophical Magazine \& Philosophical Magazine Letters \\
\hline Manuscript ID: & TPHM-05-May-0195.R1 \\
\hline Journal Selection: & Philosophical Magazine \\
\hline Author: & 05-Jul-2005 \\
\hline Complete List of Authors: & $\begin{array}{l}\text { Feuerbacher, M.; Forschungszentrum Jülich, Institut für } \\
\text { Festkörperforschung } \\
\text { Heggen, Marc; Forschungszentrum Jülich, Institut für } \\
\text { Festkörperforschung }\end{array}$ \\
\hline Keywords: & plasticity, defects \\
\hline Keywords (user supplied): & complex metallic alloys, metadislocations \\
\hline
\end{tabular}

\section{今scholaroNE \\ Manuscript Central}


On the concept of metadislocations in complex metallic alloys

By M. FEUERBACHER and M. HEGGEN,

Institut für Festkörperforschung, Forschungszentrum Jülich GmbH, 52425 Jülich, Germany

Metadislocations are a novel type of structural defect in complex metallic alloys.

We present an experimental characterization of metadislocation loops in $\xi$ '-Al-Pd-

Mn by means of transmission electron microscopy. Furthermore we discuss the mode of metadislocation motion (glide vs. climb) and the geometrical structure of metadislocations in other orthorhombic complex metallic alloys.

Keywords: Complex metallic alloys; Metadislocations; Defects; Plasticity

\section{Introduction}

Complex metallic alloys (CMAs) represent a newly emerging field of materials science. These materials are characterized by a complex atomic structure and generally possess large lattice constants. The number of atoms per unit cell is high, ranging from a hundred to some thousands. The local atomic order in CMAs is characterized by a cluster substructure. Frequently, icosahedral-symmetric atomic arrangements, e.g. Mackay or Bergman clusters are found [1].

As direct consequence of the salient type of local order, CMAs possess a variety of physical properties, which significantly differ from those in ordinary metals (see e.g. [2]). In this paper we will address a recently discovered type of structural defect, the metadislocation. Metadislocations have been firstly observed in the orthorhombic phase $\xi$ '-Al-Pd-Mn [3] and for the first time provide a physically evident plastic deformation mode for large-unit-cell 
materials. The different types of metadislocation and their structural properties have been investigated in some detail experimentally [4] and theoretically [5]. It is the aim of the present paper, to discuss some hitherto unregarded aspects of metadislocations, their mode of movement, and to examine the possibility of metadislocations in phases other than $\xi^{\prime}$-Al-Pd$\mathrm{Mn}$.

\section{Metadislocations in $\xi$ '-Al-Pd-Mn: Recall of previous results}

६'-Al-Pd-Mn has an orthorhombic lattice (space group Pnma), the unit cell contains about 320 atoms. The cell parameters are $a=23.89 \AA, b=16.56 \AA$, and $c=12.56 \AA$ [6]. Viewed along the $\left[\begin{array}{lll}0 & 1 & 0\end{array}\right]$ direction, a hexagon lattice composed of two types of flattened hexagon arranged in alternating orientations can be used for a schematic representation of the ideal structure as shown in Fig.1 (a). A comparison with the atomic structure of the $\xi$ '-phase shows that the vertices of the hexagons are decorated with 52-atom clusters of local icosahedral order, the so called Pseudo Mackay Clusters.

Klein et al. have discovered a salient type of defect in the $\xi^{\prime}$-Al-Pd-Mn structure which they have termed phason lines [7]. These are linear defects extended along [ $\left[\begin{array}{lll}0 & 1 & 0\end{array}\right]$ represented in the hexagon lattice by additional tiles, a banana-shaped polygon with an attached pentagon (hatched tiles in Fig. 1 b). They can move in the structure along the [ $\left[\begin{array}{lll}0 & 0 & 1\end{array}\right]$ direction by local atomic rearrangements, represented as vertex flips in the hexagon lattice. Phason lines preferentially line up along the $\left[\begin{array}{lll}1 & 0 & 0\end{array}\right]$ direction, forming a $\left(\begin{array}{lll}0 & 0 & 1\end{array}\right)$ plane. Such planes are referred to as phason planes.

$\xi^{\prime}-\mathrm{Al}-\mathrm{Pd}-\mathrm{Mn}$ is the basic phase of a family of related structures with equal $a$ - and $b$ - but larger $c$-lattice parameters. The most prominent of these possesses a $c$-parameter of $58 \AA$ and has 
been termed $\psi-\mathrm{Al}-\mathrm{Pd}-\mathrm{Mn}$. The structure of $\psi-\mathrm{Al}-\mathrm{Pd}-\mathrm{Mn}$ can be represented in terms of flattened hexagons as for the case of $\xi^{\prime}$-Al-Pd-Mn with additional periodic $\left[\begin{array}{lll}0 & 0 & 1\end{array}\right]$ stacking of phason planes in distances of $29.0 \AA$ (Fig. 1 b). Other related structures with $c=32.4 \AA$, 44.9 $\AA$, and 70.1 $\AA$ have been reported [8].

Metadislocations represent a novel type of defect addressing a basic issue of the plasticity of large-unit-cell materials. They are partial $\left[\begin{array}{lll}0 & 0 & 1\end{array}\right]$ edge dislocations, possessing, on the one hand, energetically favourable short Burgers-vector magnitudes. On the other hand, glide motion of these partials is possible without alteration of the structure (e.g. the introduction of a stacking fault or an antiphase boundary). This is due to the particular accommodation of the dislocation core into the ideal structure involving phason planes. The associated phason planes are a vital constituent of the metadislocation and hence have to be dragged along with the dislocation upon movement of the latter (see section 4). Fig. $1 \mathrm{c}$ shows a schematic view of a metadislocation with a Burgers vector length of $1.83 \AA$. The dark-grey polygon which represents the dislocation core is accommodated to the $\xi^{\prime}$-Al-Pd-Mn structure by six associated phason half planes. A geometrical description of this accommodation mechanism is given by Klein et al. [3]. Other types of metadislocation with [0 00 1] Burgers vector of 0.70 $\AA$, $1.13 \AA, 2.96 \AA$, and $4.80 \AA$ in magnitude, associated with 16, 10, 4, and 2 phason half planes, respectively, were reported by Klein et al. [4]. Feuerbacher et al. [9] have demonstrated that for appropriate deformation geometries, metadislocations mediate the plastic deformation behaviour of $\xi^{\prime}$-Al-Pd-Mn. Fig. 2 shows a transmission electron microscopy image of a metadislocation with six associated phason planes in an otherwise perfect matrix of $\xi^{\prime}-\mathrm{Al}-\mathrm{Pd}-\mathrm{Mn}$ structure.

\section{Metadislocation loops}


Dislocations are one-dimensional structural defects representing the boundary of an area over which a given displacement has occurred. Hence, a dislocation line evidently cannot end within an otherwise perfect region of crystal. It must terminate e.g. at a free surface, another dislocation line, or a grain boundary [10]. Generally, a dislocation in a perfect and infinite crystal is a closed loop. Sole segments observed experimentally are parts of loops arbitrarily cut out by the specimen surfaces. All current reports on metadislocations have focused on [0 1 0] segments of the corresponding loops. However, the above considerations hold for metadislocations as well, and hence the question for the other segments, completing loops with the $\left[\begin{array}{lll}0 & 1 & 0\end{array}\right]$ segments must be approached in order to obtain a complete view.

We have carried out TEM investigations on deformed $\psi$-Al-Pd-Mn single crystals grown by the Bridgman technique. The material was deformed in compression to $8 \%$ strain at $725{ }^{\circ} \mathrm{C}$. Fig. 3 a shows a two-beam bright-field image taken close to the $\left(\begin{array}{lll}1 & 0 & 3\end{array}\right)$ zone axis. This direction makes an angle of $90^{\circ}$ with the $\left[\begin{array}{lll}0 & 1 & 0\end{array}\right]$ direction and of $32.5^{\circ}$ with the $\left[\begin{array}{lll}0 & 0 & 1\end{array}\right]$ direction. The reflection $\boldsymbol{g}=\left(\begin{array}{lll}\overline{8} & 4 & 3\end{array}\right)$ was used for imaging. The image shows a high density of elongated dislocation loops. Fig. 3 b shows the same specimen area imaged using $\boldsymbol{g}=\left(\begin{array}{lll}0 & 8 & 0\end{array}\right)$. The contrast of the dislocation loops is extinct under these conditions. We also observed contrast extinction with all other reflections perpendicular to [ $\left[\begin{array}{lll}0 & 0 & 1\end{array}\right]$. With these results we can conclude that the Burgers vector of the dislocations loops imaged is parallel to the $\left[\begin{array}{lll}0 & 0 & 1\end{array}\right]$ direction.

By means of tilting experiments we have determined the habit planes of the dislocation loops as $\left(\begin{array}{lll}0 & 0 & 1\end{array}\right)$ planes. The long segments in Fig. 3 a are parallel to the $\left[\begin{array}{lll}0 & 1 & 0\end{array}\right]$ direction and the short segments (one is marked in the figure) to the $\left[\begin{array}{lll}1 & 0 & 0\end{array}\right]$ direction.

Due to the limited specimen-tilting angle in the TEM, it is experimentally impossible to turn the specimen as far as $90^{\circ}$ to the $\left(\begin{array}{lll}0 & 1 & 0\end{array}\right)$ zone axis in order to confirm directly that the loops observed correspond to metadislocations. However, we have strong evidence that this is so. First, the density of line segments along the $\left[\begin{array}{lll}0 & 1 & 0\end{array}\right]$ direction in Fig. 3 is consistent with the 


\section{Mode of metadislocation motion}

As yet, not direct observation of metadislocation motion, e.g. by in-situ tensile tests in a transmission electron microscope could be made. Therefore the question of the mode of metadislocation movement, in particular whether metadislocations motion takes place by glide or climb can to date not be resolved experimentally. We will present, however, in the following a number of arguments, which strongly indicate that metadislocations move by climb rather than glide.

The first argument, in fact, has already been presented in section 3, where it was experimentally demonstrated that metadislocation loops are pure edge loops. Edge loops can 
only expand, i.e. contribute to straining of the sample, if their segments move by climb. Glide motion of the segments would merely lead to unaltered movement of the loop though the structure without any effect of straining. Therefore, metadislocations can only mediate plastic deformation if they move by climb. Evidence for the fact that metadislocations indeed mediate plastic strain has been found in compression tests, which showed a significant increase of metadislocation density upon plastic deformation.

For an argument against glide motion, let us introduce a new tiling for the description of the phason-lines. Fig. 4 a shows the same metadislocation as in Fig. $1 \mathrm{c}$ but now the two tiles representing the phason lines have been replaced by three irregular hexagons. (hatched area). This tiling is not very well suited to represent the structure of the material itself, since the additional vertices do not reflect positions of Mackay-type cluster centres, but on the other hand, it does have the advantage that the movement of every single vertex of the hexagon lattice can be traced during movements of phason lines or metadislocations.

Using this new tiling, Fig. 4 b schematically shows a glide step (upward movement along [0 0 1]) of a metadislocation by one $c$-lattice constant. The initial position of all tiles is given by the black lattice (which is hence identical to Fig. 4 a), the tile representing the initial metadislocation-core position is shaded grey. The final positions are given by the grey lattice. Only those tiles of the final lattice, the position or shape of which is altered, are shown. It is clearly seen that a very high number of vertex flips is necessary to proceed from the initial to the final position. In fact, the number of vertex flips is approximately proportional to the area of the phason planes. This is simply due to the fact that during glide motion, the phason planes associated with the metadislocation have to be dragged through the material with the core.

Fig. 4 c schematically shows a climb step (movement along $\left[\begin{array}{lll}1 & 0 & 0\end{array}\right]$ to the left) of a metadislocation by one $a$-lattice constant. Again, the initial tiles are given by black and the final tiles by grey lines. It is clearly seen that for a climb step a much smaller number of 
vertex jumps is necessary. The number of necessary vertex jumps is now limited to the core region and hence the number of vertex jumps can be numeralized no matter how far the associated phason planes are extended. Since each vertex jump physically corresponds to a number of local atomic jumps (see [11] for a correlation of atom jumps and vertex jumps in the original tiling) it is directly evident that climb motion is connected with much less effort concerning local atomic rearrangement than glide motion.

Note however, that long-range atomic transport is necessary for the climb process because, in contrast to the glide process, it is a non-conservative mode of dislocation motion. Depending on the direction of movement, either atoms or vacancies have to be moved to the dislocation core region. This fact is also directly reflected in our schematics: In Fig. 4 b, showing the glide step, each newly occurring vertex in the final hexagon lattice has a corresponding vertex in the initial lattice. This is not the case for the climb step shown in Fig. 4 c. In present depiction, the final lattice has 28 new vertices which have to be balanced against 24 vertices in the initial lattice. That is, four new vertices have to be created, or, in terms of atom positions, a number of atoms have to be transported to the core region (the exact number of which, of course, can only be calculated on the basis of an atomic model of the metadislocation core and the phason lines).

The necessity of atomic transport is not a principal objection against climb motion. Climb motion, as a non-conservative process, requires long-range atomic motion no matter whether it takes place in a complex metallic alloy, a simple metal or even a quasicrystal. For the latter two types of material it has been demonstrated that climb takes place and can represent the primary mode of dislocation motion $[12,13]$. The condition for the preference of climb motion for the present material, according to the above considerations is apparent: If long-range atomic transport to the metadislocation core is energetically less costly than the numerous local atomic jumps necessary for the movement of the associated phason planes, climb should be preferred. Since the material is only ductile at high temperatures, where diffusive motion is 
greatly facilitated, this condition may easily be fulfilled. The situation may, however, be completely different if metadislocation networks, in which only short phason planes exist, are involved in plasticity [14].

\section{Metadislocations in other structures}

Metadislocations have been reported in the literature up to now only in $\xi^{\prime}-\mathrm{Al}-\mathrm{Pd}-\mathrm{Mn}$ and the related phase $\psi$-Al-Pd-Mn (see e.g. [3,4]). $\xi^{\prime}$-Al-Pd-Mn is a member of a class of phases, which are frequently referred to as $\varepsilon$-phases. These phases have orthorhombic structures and have Mackay-type clusters as basic structural elements.

$\varepsilon$-phases exist in numerous Al-based alloy systems. The basic phase is frequently referred to as $\mathrm{Al}_{3} \mathrm{Pd}$. To be more precise, it exists in the compositional range of about 72 to 76 at.\% $\mathrm{Al}$. The $\varepsilon$-phases make up a phase family, the members of which possess similar $a$ - and $b$-lattice parameters and various $c$-lattice parameters, which are related by powers of the golden mean $\tau=1 / 2(\sqrt{5}+1)$. The phases are frequently referred to as $\varepsilon_{\mathrm{i}}$, where $\mathrm{i}=6,16,22,28, \ldots$ refers to the index $\left(\begin{array}{lll}0 & 0\end{array}\right)$ of the first strong reflection corresponding to the interplanar spacing of about $0.2 \mathrm{~nm}$. The basic phase $\mathrm{Al}_{3} \mathrm{Pd}$ has ternary extensions in a number of systems such as $\mathrm{Al}-\mathrm{Pd}-$ $(\mathrm{Mn}, \mathrm{Fe}, \mathrm{Co}, \mathrm{Ru}, \mathrm{Re}, \ldots)$ at approximately constant Al-content up to several percent into the ternary range. Al-Pd-Fe, for example, exists with up to 10 at.\% Fe. $\varepsilon$-phases also exist in $\mathrm{Al}_{3} \mathrm{Rh}$ and the ternary extensions $\mathrm{Al}-\mathrm{Rh}-(\mathrm{Cu}, \mathrm{Ni}, \ldots)$. The $\xi^{\prime}-$ and $\psi-\mathrm{Al}-\mathrm{Pd}-\mathrm{Mn}$ phases, in these terms, are referred to as $\varepsilon_{6}$ and $\varepsilon_{28}$, respectively.

All $\varepsilon_{6}$-phases can be described by a lattice of alternatingly oriented flattened hexagons as shown in Fig. 1 a. All higher phases $\varepsilon_{\mathrm{i}}$ can be described by periodic arrangements of phason planes as shown in Fig. 2 b. The figure corresponds to $\varepsilon_{28}$, all other phases are represented by 
larger or smaller distances between the phason planes [15]. As a direct consequence, metadislocations can exist in all $\varepsilon$-phases and they can all be described by a schematic as given by Fig. 1 c. The first observation of a metadislocation in an $\varepsilon$-phase other than $\xi^{\prime}$ - and $\Psi$-Al-Pd-Mn has recently been made in $\varepsilon-\mathrm{Al}-\mathrm{Pd}-\mathrm{Fe}[16]$.

Let us now consider another important set of orthorhombic phases, which are based on the binary phase $\mathrm{Al}_{3} \mathrm{Mn}$. The structure of $\mathrm{Al}_{3} \mathrm{Mn}$ was firstly approached by Taylor [17] and therefore this phase and the phases derived from it are referred to as T-phases. The basic Tphases has lattice parameters of $a=14.8 \AA, b=12.4 \AA$, and $c=12.6 \AA$ [17]. The binary $\mathrm{Al}_{3} \mathrm{Mn}$ phase has known ternary extensions into the systems $\mathrm{Al}-\mathrm{Mn}-(\mathrm{Pd}, \mathrm{Co}, \mathrm{Cr})$.

The T-phases can also be described in terms of a lattice constructed using alternatingly oriented hexagons. Such a lattice is shown in Fig. 5 a. For the construction, a different type of hexagon than for the $\varepsilon$-phases is used, which we term "elongated" hexagon. The obtuse angle in the hexagons used for the description of the $\varepsilon$ - and the T-phases is equal and corresponds to the internal angle of a regular pentagon. Klein et al. [18] have considered the T-phase in the system Al-Mn-Pd and have shown that, in complete analogy with the $\varepsilon$-phase in Al-Pd-Mn, phason lines exist. The phason lines are represented by crown-shaped tiles in the hexagon lattice (hatched area in Fig. 5 b). Accordingly, structures in analogy to $\varepsilon_{\mathrm{i}}$ can be constructed as shown in Fig. 5 b. The figure shows a structure corresponding to $\varepsilon_{28}$ (or $\Psi-A l-P d-M n$ ). Structures of this type have indeed been observed in Al-Cr-Fe [19] and $\mathrm{Ga}_{7} \mathrm{Mn}_{5}$ [20] and do most probably exist in the system Al-Mn-Pd [21]. Other structures with different $c$-lattice parameter can be accordingly constructed introducing larger or smaller spacing between the phason planes.

With this basis, all geometrical requirements for the construction of metadislocations in the Tphases are provided. Therefore, we propose for the T-phases a metadislocation structure as schematically represented in Fig. 5 c. The figure shows a metadislocation with six associated 
phason planes. Its core is given by the dark grey tile. The Burgers-vector length can be calculated as $b_{T}=c / \tau^{4}$. Using the lattice parameter $c=12.59 \AA$ of T-Al-Mn-Pd [18] we obtain a Burgers vector length of $1.84 \AA$. In complete analogy with $\xi^{\prime}-$ Al-Pd-Mn, a sequence of metadislocations of this type can exist, with two, four, six, ten, and 16 associated phason planes [4]. The corresponding Burgers vector lengths are $4.81 \AA$, $2.97 \AA, 1.84 \AA, 1.14 \AA$, and $0.70 \AA$, respectively. Note that these Burgers vector lengths are related by powers of the golden mean $\tau$. Metadislocations, as schematically represented in Fig. 5 c, may exist in all Tphases, in the basic phases as represented by Fig. 5 a as well as in the derived phases as represented by Fig. 5 b. Metadislocations in T-phases are also discussed by Engel and Trebin [22] (this volume).

Let us consider a third set of orthorhombic phases, which are commonly referred to as $\mathrm{Al}_{13} \mathrm{M}_{4}$ phases. A well known representative of this phase is $\mathrm{Al}_{13} \mathrm{Co}_{4}$ [23], in $\mathrm{Al}_{13} \mathrm{Fe}_{4}$ an orthorhombic high-temperature phase isostructural to $\mathrm{Al}_{13} \mathrm{Co}_{4}$ exists (which is a replacive high-temperature variant of the monoclinic low-temperature phase) [24]. These structures can be described using a tiling constructed by a regular pentagons and a rhombus as shown in Fig. 6 a. Proceeding in full analogy to the preceding cases, we construct phason lines by introducing an additional tile, a boat shaped heptagon*. The phason lines can be arranged along the $\left[\begin{array}{lll}0 & 1 & 0\end{array}\right]$ direction, forming $\left(\begin{array}{lll}0 & 0 & 1\end{array}\right)$ planes. Periodic stacking of these planes leads to the hypothetical structure shown in Fig. 6 b. Variants with different $c$-lattice parameters, of course, can be constructed by introducing larger or smaller spacing between the phason planes. The so constructed elements can be used to build another type of metadislocation for the $\mathrm{Al}_{13} \mathrm{M}_{4}$ phases. Fig. $6 \mathrm{c}$ shows a hypothetical metadislocation with six associated phason planes. Its core is given by the dark grey tile. The Burgers-vector length can be calculated as $b_{A M}=b / \tau^{4}$. Using the lattice parameter $b=12.34 \AA$ of orthorhombic $\mathrm{Al}_{13} \mathrm{Co}_{4}$ [23] we obtain

\footnotetext{
* Phason lines in these structures can alternatively be described in terms of irregular hexagons.
} 
a Burgers vector length of $1.80 \AA$. Again, a sequence of metadislocations of this type can be constructed, with two, four, six, ten, and 16 associated phason planes. The corresponding Burgers vector lengths (again related by powers of the golden mean $\tau$ ) are $4.71 \AA, 2.91 \AA$, $1.80 \AA$, $1.11 \AA$, and $0.69 \AA$, respectively. Such metadislocations may exist in the basic $\mathrm{Al}_{13} \mathrm{M}_{4}$ phases as well as in the derived phases with other $b$-lattice parameters.

Surely, the construction of metadislocations for structures other than $\xi^{\prime}-$ or $\Psi$-Al-Pd-Mn is rather speculative. The considerations made above merely show that the concept of metadislocations is not limited to a single phase. It can be transferred to other structures and, from a purely geometric point of view, leads to possible metadislocation structures in the corresponding phases. It is of course not said that the proposed metadislocations do exist in these materials. This will depend on many, partly unknown factors, such as the exact coexistence of phases in the phase diagram (which will determine whether or not the formation of phason planes and their periodic arrangement is energetically favourable), the energy cost of stacking faults (which will determine whether or not competing, deformation mechanisms based on conventional partials are favourable), etc.

\section{Acknowledgements}

The authors thank S. Balanetskyy and B. Grushko for inspiring discussions. Carsten Thomas and Marita Schmid are thanked for performing the single-crystal growth.

\section{References}

[1] N. Tamura, Phil. Mag. A 76, 337 (1997). 
[2] K. Urban and M. Feuerbacher, J. Non-Cryst. Sol. 334, 143 (2003).

[3] H. Klein, M. Feuerbacher, P. Schall, and K. Urban, Phys. Rev. Lett. 82, 3468 (1999).

[4] H. Klein and M. Feuerbacher, Philos. Mag. 83, 4103 (2003).

[5] M. Engel and H.R. Trebin, Philos. Mag. (2005), in press.

[6] M. Boudard, H. Klein, M. DeBoissieu, M. Audier, and H. Vincent, Philos. Mag. A74, 939 (1996).

[7] H. Klein, M. Audier, M. Boudard, M. de Boissieu, L. Beraha, and M. Duneau, Philos. Mag. A. 73, (1996) 309.

[8] M. Yurechko, B. Grushko, T. Ya. Velikanova, and K. Urban, in Phase Diagrams in Materials Science, edited by T. Ya. Velikanova (Materials Science International Services, Stuttgart, 2004).

[9] M. Feuerbacher, H. Klein, and K. Urban, Philos. Mag. Lett. 81, 639 (2001).

[10] J.P. Hirth and J.Lothe, Theory of Dislocations (Krieger, Malabar, 1992), p. 24.

[11] L. Beraha, M. Duneau, H. Klein, M. Audier, Philos. Mag. A 76, 587 (1997).

[12] G. Edelin and J. P. Poirier, Philos. Mag. 28, 1203 (1973).

[13] D. Caillard, G. Vanderschaeve, L. Bresson, and D. Gratias, Phil. Mag. A 80, 237 (2000).

[14] M. Heggen and M. Feuerbacher, this volume.

[15] M. Feuerbacher, Ch. Thomas, and K. Urban, in Quasicrystals, edited by H.R. Trebin (Wiley-VCH, Weinheim, 2003).

[16] S. Balanetskyy, private communication.

[17] M.A. Taylor, Acta Cryst. 14 (1961) 84.

[18] H. Klein, M. Boudard, M. Audier, M. De Boissieu, H. Vincent, L. Beraha, and M. Duneau, Philos. Mag. Lett. 75, (1997) 197.

[19] V.Demange, J. Ghanbaja and J.M. Dubois, this volume.

[20] J.S. Wu, S.P. Ge and K.H. Kuo. Phil. Mag. A. 79, 1787 (1999).

[21] S. Balanetskyy and M. Feuerbacher, to be published. 
[24] M. Engel and H.-R. Trebin, this volume.

[23] J. Grin, U. Burkhardt, M. Ellner, and K. Peters. J. All. Comp. 206, 243 (1994).

[24] M. Ellner, Acta Cryst. B51, (1995) 31. 


\section{Figure captions}

Fig. 1: Schematic representation of the ideal structure of $\xi^{\prime}-\mathrm{Al}-\mathrm{Pd}-\mathrm{Mn}$ (a) and $\Psi-\mathrm{Al}-\mathrm{Pd}-\mathrm{Mn}$ (b) and a metadislocation with six associated phason planes. The grey boxes indicate the unit cells of the respective phases. A single phason line is hatched in (b)

Fig. 2: Transmission electron micrograph of a metadislocation with ten associated phason half planes.

Fig. 3: Transmission electron micrographs of metadislocation loops. Loops in contrast with $\boldsymbol{g}=\left(\begin{array}{lll}\overline{8} & 4 & 3\end{array}\right)(\mathrm{a})$; contrast extinction with $\boldsymbol{g}=\left(\begin{array}{lll}0 & 8 & 0\end{array}\right)(\mathrm{b})$. The arrow points at one of the $\left[\begin{array}{lll}1 & 0 & 0\end{array}\right]$ segments.

Fig. 4: Schematic representation of a metadislocation (a) using a modified hexagon lattice. (b): glide step; (c) climb step. The initial positions are shown by black lines, the altered final positions are shown by grey lines. See text.

Fig. 5: Ideal structure of the T-phase (a), the related structure based on periodically stacked phason planes (b), and a metadislocation with six associated phason planes. The grey boxes indicate the unit cells of the respective phases. A single phason line is hatched in (b)

Fig. 6: Ideal structure of the $\mathrm{Al}_{13} \mathrm{M}_{4}$ phase (a), the related structure based on periodically stacked phason planes (b), and a metadislocation with six associated phason planes. The grey boxes indicate the unit cells of the respective phases. A single phason line is hatched in (b) 


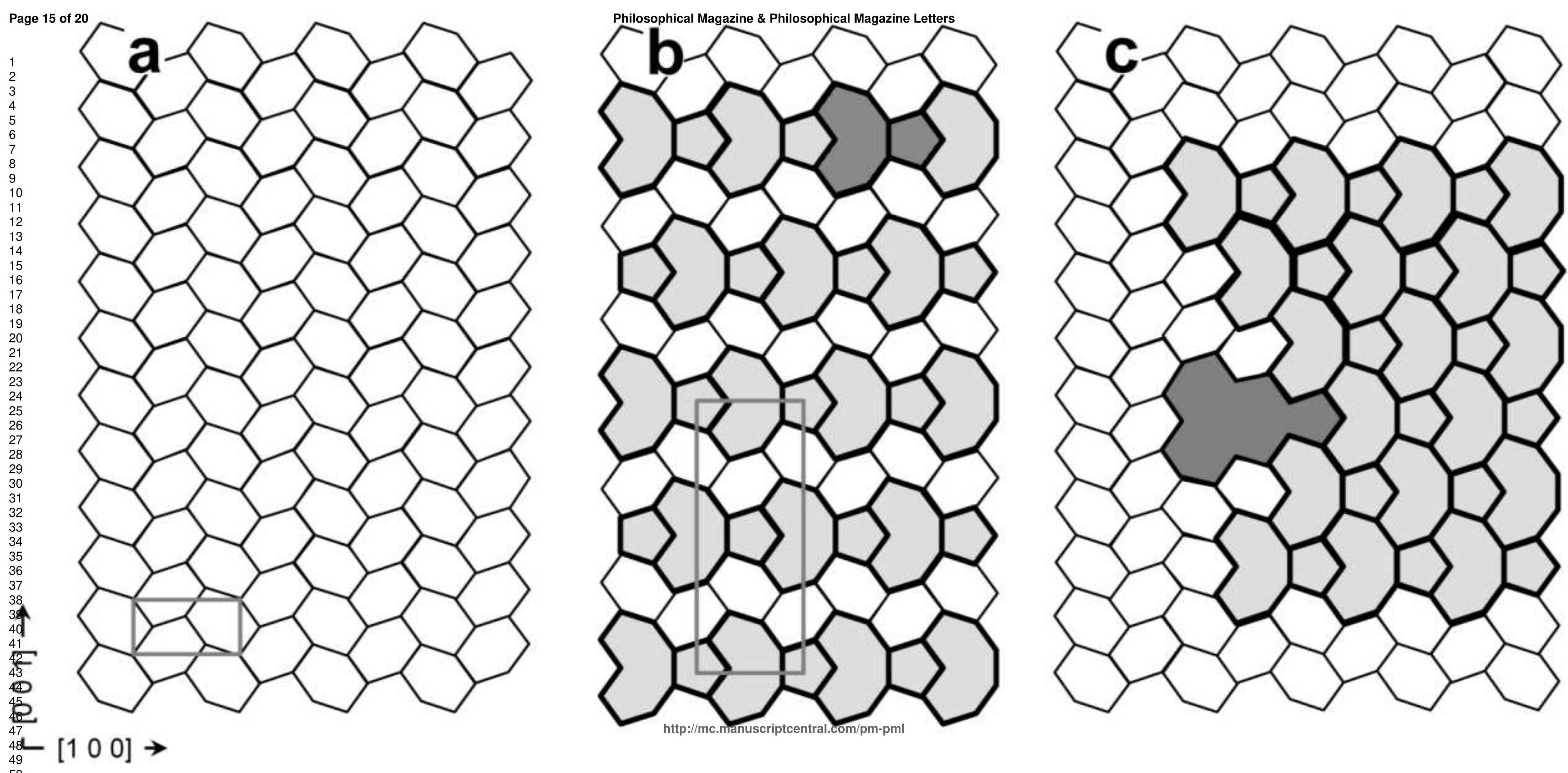




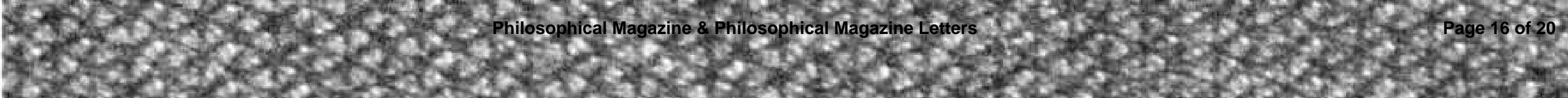

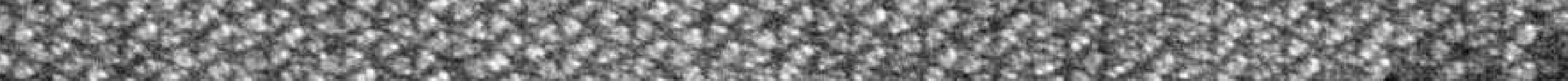

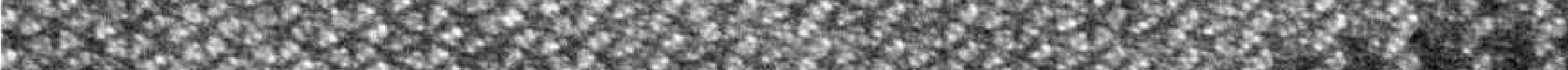

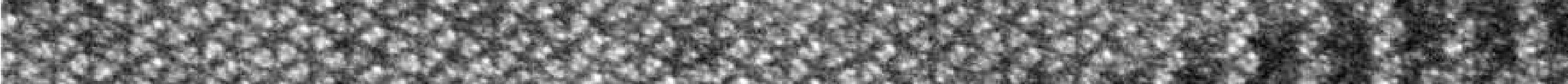

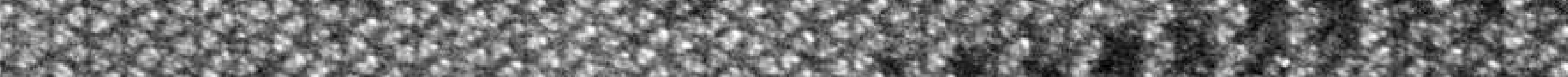

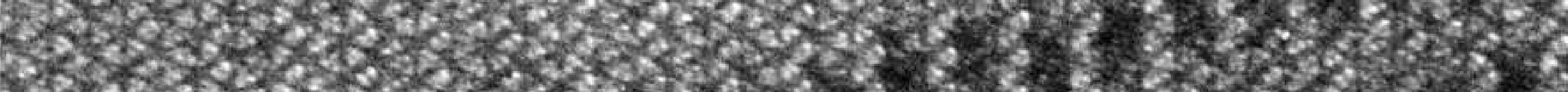

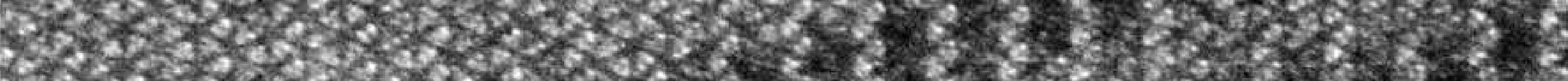

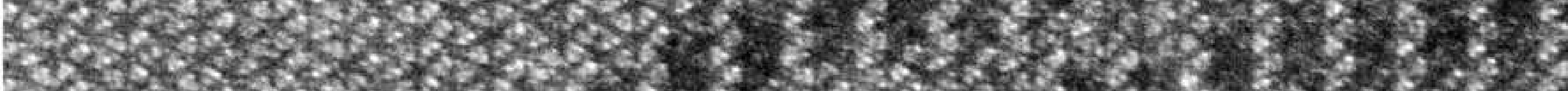

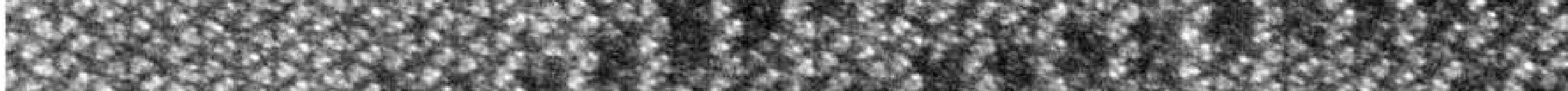

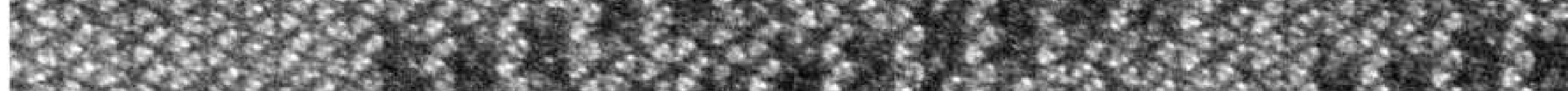

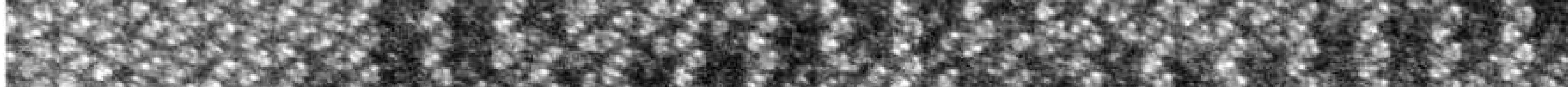

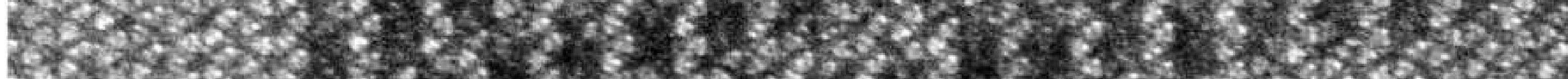

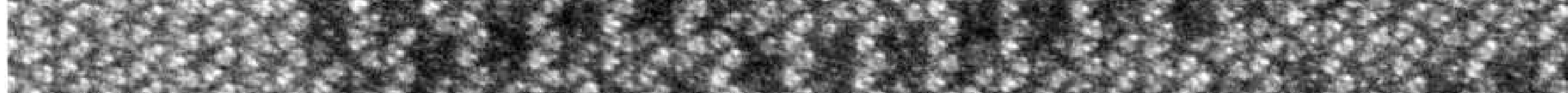

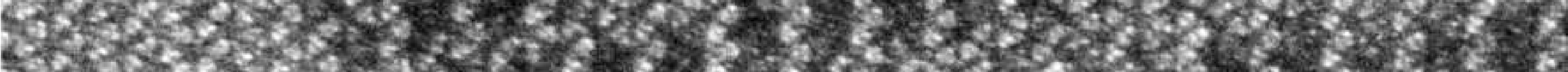

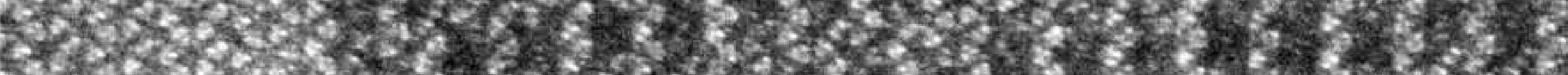

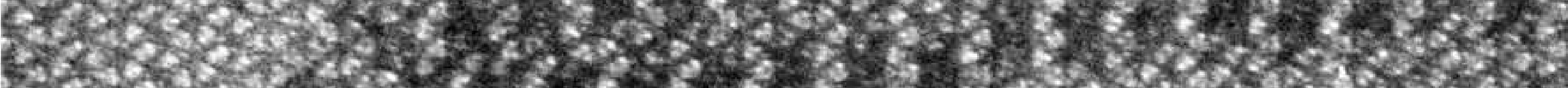

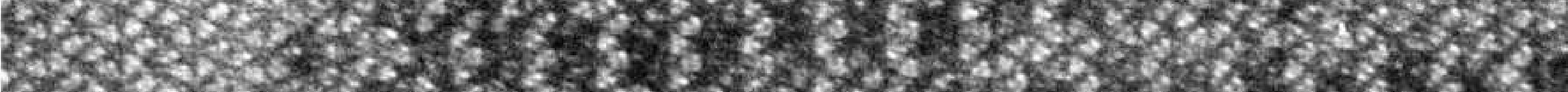

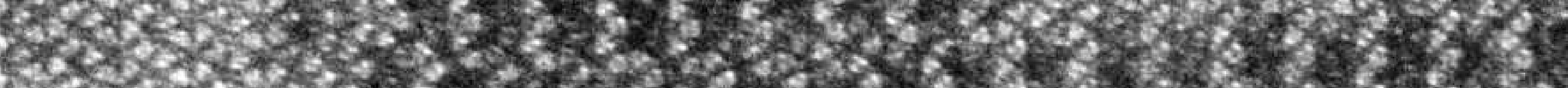

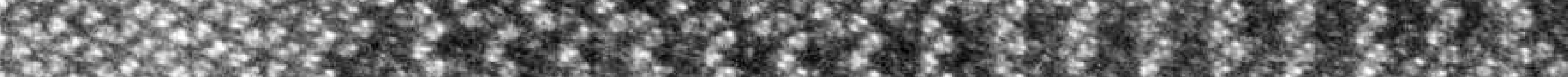

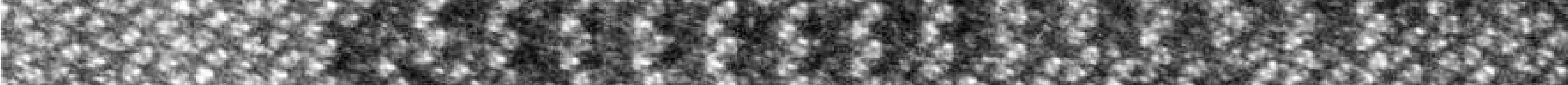

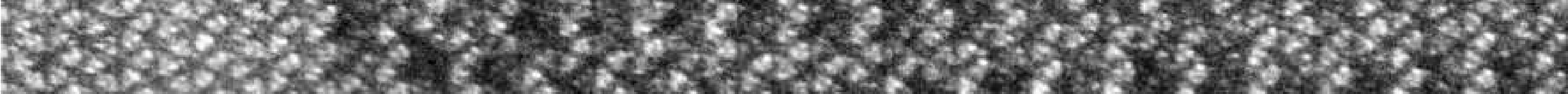

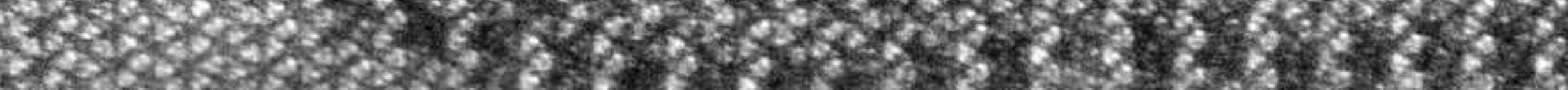

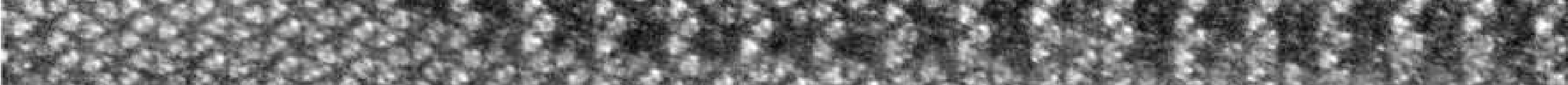

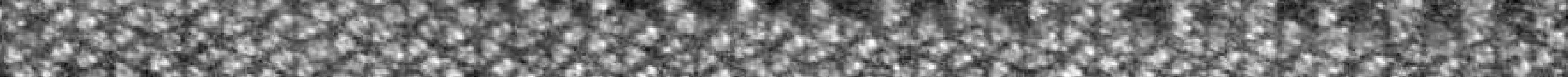

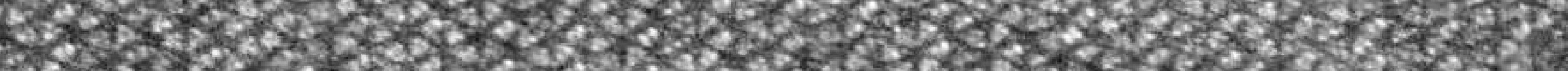

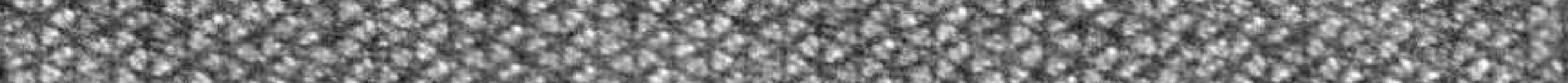

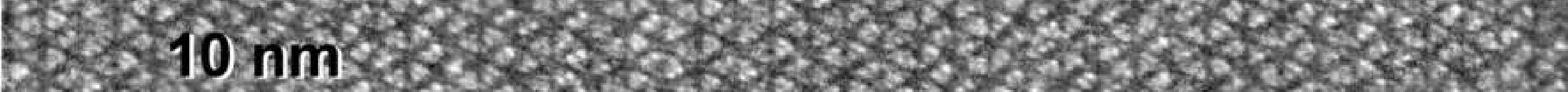

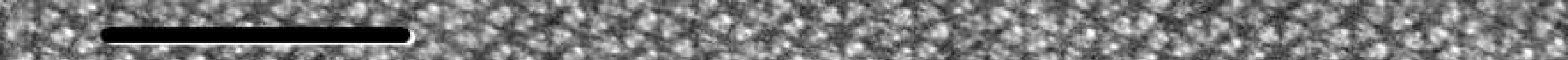

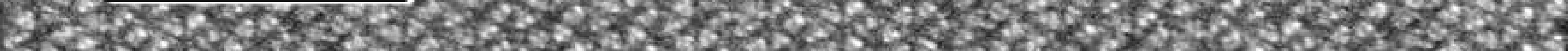

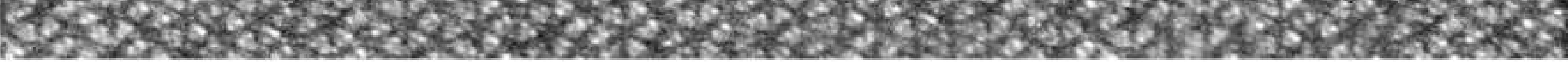




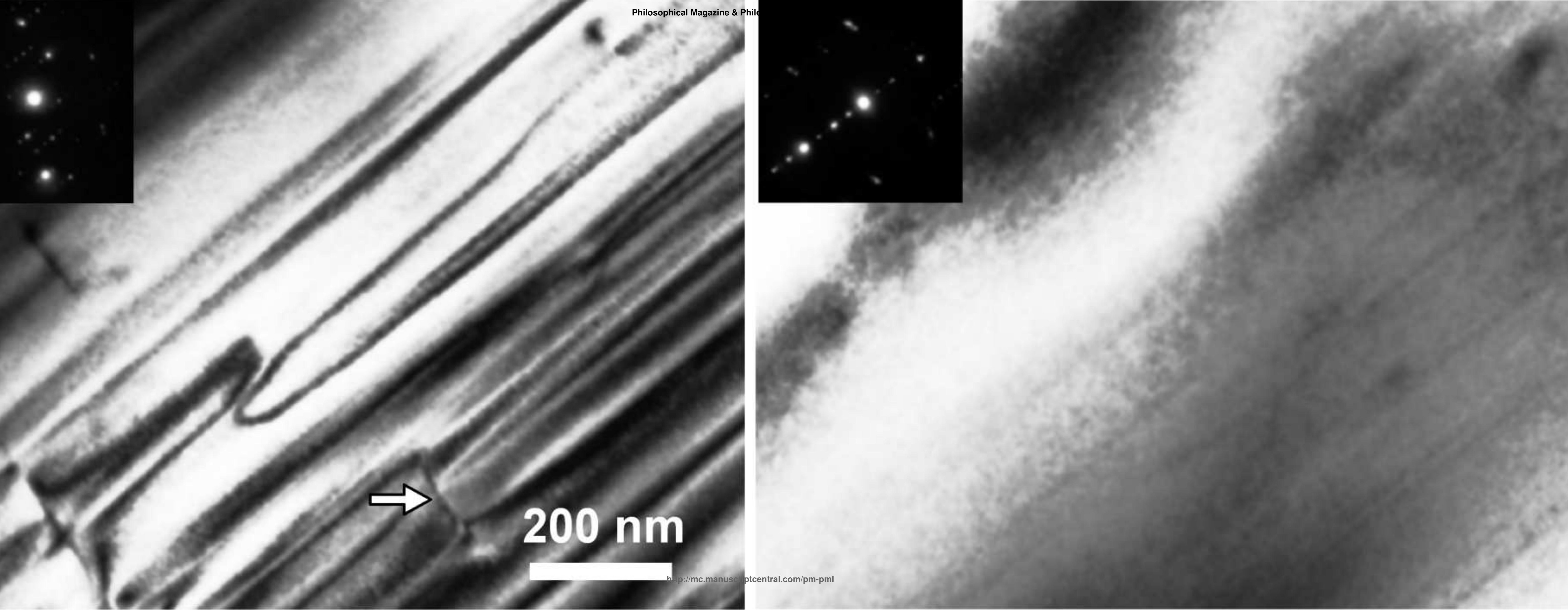




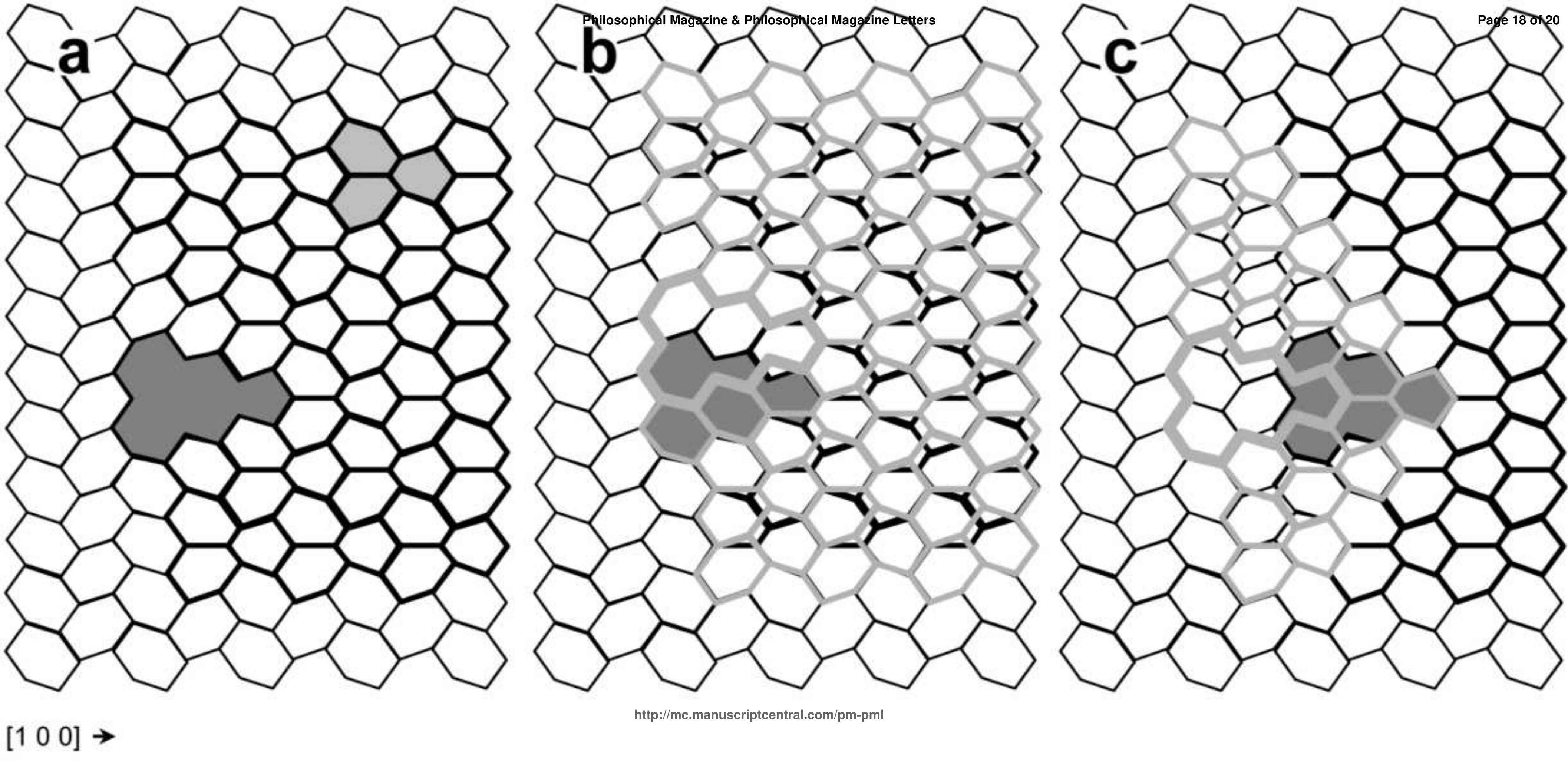




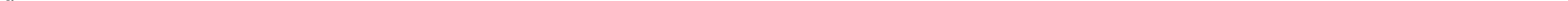




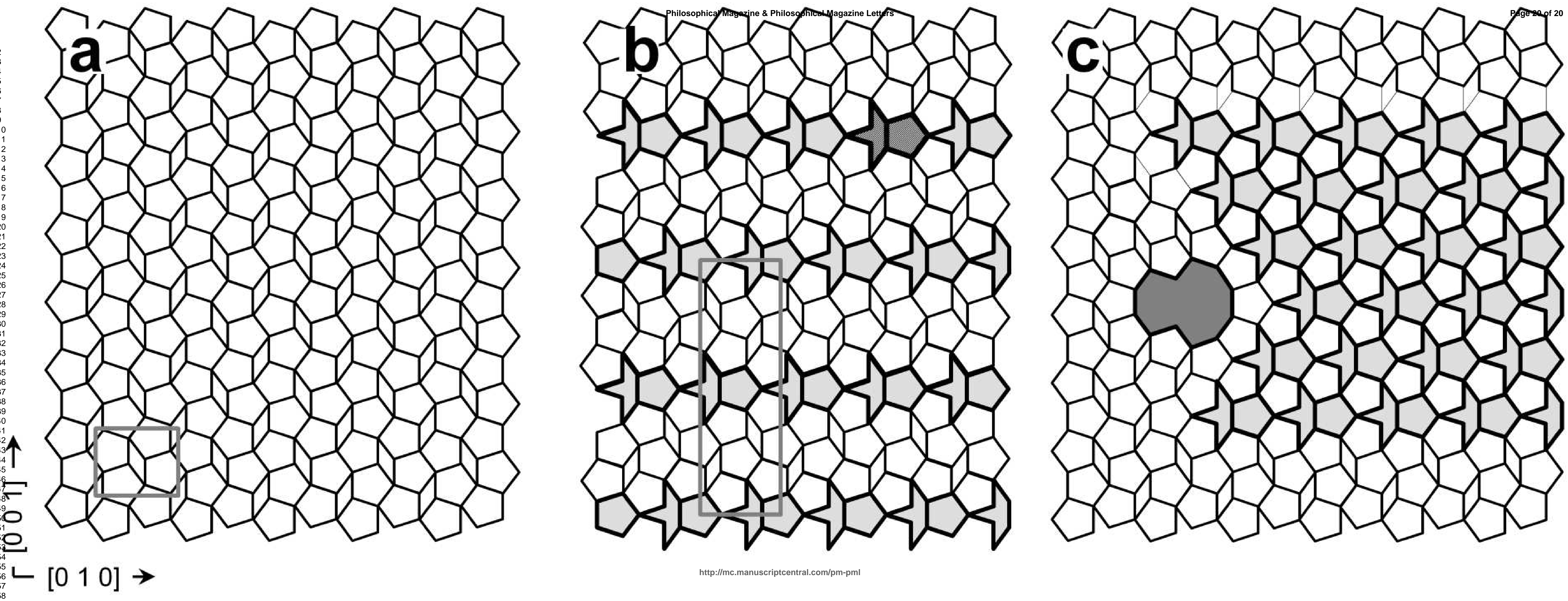

